\title{
A GENERALIZED CANTOR THEOREM
}

\author{
A. M. GLEASON AND R. P. DILWORTH
}

A well known theorem of Cantor asserts that the cardinal of the power-set of a given set always exceeds the cardinal of the original set. An analogous result for sets having additional structure is the well known theorem that the set of initial segments of a well ordered set always has order type greater than the original set. These two theorems suggest that there should be a similar result for general partially ordered sets. In formulating such a theorem an extension to partially ordered sets of the notion of an initial segment of a well ordered set is required. Of the several possibilities for this choice, the most natural one is the concept of an order ideal. If $P$ is a partially ordered set with order relation $\leqq$, then a subset $I$ of $P$ is an order ideal if $a \leqq b \in P$ implies $a \in P$. The set $g(P)$ of all order ideals of $P$ is easily seen to be a complete partially ordered set when ordered by set inclusion, since the union and intersection of any set of order ideals is again an order ideal. Note that the empty set is specifically included among the order ideals.

The general theorem can then be formulated as follows:

Theorem A. If $P$ is any partially ordered set and $g(P)$ is the set of all order ideals of $P$, then $g(P)$ is not order isomorphic to any subset of $P$.

The authors found two independent proofs of this theorem which upon analysis indicated that an even stronger theorem holds.

ThEOREM B. Let $P$ be a partially ordered set and let $\mathscr{S}(P)$ denote the set of order ideals of $P$. Then if $\phi$ is a one-to-one map of $\mathscr{g}(P)$ into $P$, neither $\phi$ nor $\phi^{-1}$ is order preserving.

In the terminology of Theorem B, Theorem A asserts that $\phi$ and $\phi^{-1}$ are not both order preserving.

The Cantor theorem follows from this result by assigning to an arbitrary set $P$ the trivial order relation (no unequal elements are comparable). Then $g(P)$ is the power set of $P$ and the inverse $\phi^{-1}$ of a one-to-one map $\phi$ of $g(P)$ into $P$ would necessarily be order preserving. On the other hand, any infinite well ordered set is a partially ordered set for which $g(P)$ and $P$ have the same cardinal.

More generally, if $P$ is complete, linearly ordered, and infinite, then $g(P)$ and $P$ have the same cardinal. If $P$ is linearly ordered but not complete (for example, the set of rationals where $g(P)$ is isomorphic

Received by the editors September 1, 1961. 
to the Cantor set) the cardinal of $g(P)$ may be greater than the cardinal of $P$.

Proof of TheOREM B. (1) $\phi$ is not order preserving. For if $\phi$ preserves order, let $g_{0}$ the set of all order ideals $I$ for which $\phi(I) \in I$ and let $A$ be the intersection of these ideals. If $I \in g_{0}$, then $A \subseteq I$ and hence $\phi(A) \leqq \phi(I) \in I$ which implies $\phi(A) \in I$. It follows that $\phi(A)$ $\in \cap g_{0}=A$ and hence $A \in g_{0}$. Consider the ideal $B=\{p \in P \mid p<\phi(A)\}$. Clearly $B \subset A$ so that $\phi(B)<\phi(A)$ and therefore $\phi(B) \in B$. But then $B \in g_{0}$ and hence $A \subseteq B$ contrary to $B \subset A$.

(2) $\phi^{-1}$ is not order preserving. For if $\phi^{-1}$ preserves order, let $g_{1}$ denote the set of all order ideals $I$ for which $\phi(I) \notin I$ and let

$$
C=\left\{p \mid p \leqq \phi(I) \text { for some } I \in g_{1}\right\} .
$$

Then $C$ is an order ideal. If $\phi(C) \notin C$, then $C \in g_{1}$; and hence $\phi(C) \in C$ by the definition of $C$. On the other hand, if $\phi(C) \in C$, then choose $I \in g_{1}$ so that $\phi(C) \leqq \phi(I)$. By the order preserving property of $\phi^{-1}$ we have $C \subseteq I$, and since $\phi(I) \in C$ it follows that $\phi(I) \in I$ contrary to the definition of $g_{1}$.

It may be noted that the argument of part (2) also holds for quasiorderings, in which case the map need not be one-to-one.

HARVARD University,

California Institute of Technology, and Institute for Defense Analyses, Princeton, New Jersey 